\title{
MODIFICACIONES DE LOS PARÁMETROS HEMODINÁMICOS Y DE LOS DISTINTOS FLUJOS VASCULARES PERIFÉRICOS EN MODELO EXPERIMENTAL PORCINO DE NEFRECTOMÍA LAPAROSCÓPICA.
}

\author{
J. Sáenz Medina, M. S. Asuero de Lis', J. Galindo Alvarez², J. Villafruela Sanz 3, C. Correa \\ Gorospe 4 , B. Cuevas Sánchez, A. I. Linares Quevedo, A. Páez Borda, J. Pascual Santos ${ }^{5}$, \\ R. Marcén Letosa ${ }^{5}$ y J. Burgos Revillab.
}

\author{
Servicio de Urología. Hospital de Fuenlabrada. Madrid. \\ 'Departamento de Cirugía. Universidad de Alcalá. Servicio de Urología. Hospital Ramón y Cajal. Madrid. \\ 2 Servicio de Anestesiología y Reanimación. Hospital Ramón y Cajal. Madrid. \\ ${ }^{3}$ Servicio de Cirugía General. Hospital Ramón y Cajal. Madrid. \\ ${ }^{4}$ Servicio de Bioquímica. Hospital Ramón y Cajal. Madrid \\ 5 Unidad de Investigación. Hospital Ramón y Cajal. Madrid. \\ oServicio de Nefrología. Hospital Ramón y Cajal. Madrid. España.
}

\begin{abstract}
Resumen.- OBJETIVO: Se ha demostrado que la hiperpresión abdominal y la utilización del neumoperitoneo con $\mathrm{CO}_{2}$, provocan cambios en los aparatos cardiovascular y respiratorio, atribuibles a dos factores: alteraciones en el gasto cardiaco (GC) e hipercarbia. Otras alteraciones derivadas de estos hechos son los cambios en las resistencias vasculares sistémicas (RVS), la tensión arterial (TA), la presión venosa central (PVC), cambios vasculares como las alteraciones en el flujo sanguíneo renal (FSR), flujo carotídeo (FAC), flujo portal (FP) y flujo de la arteria hepática (FAH). Nuestro objetivo es analizar las modificaciones hemodinámicas producidas por el neumoperitoneo sobre los FSR, FC, FP y FAH en modelo experimental porcino.
\end{abstract}

\footnotetext{
Javier Sáenz Medina Lope de Vega, 2. Portal 5 - 1ㅡㅗ Pozuelo de Alarcón. 28223 Madrid. (España) javiersaenzmedina@yahoo.es Trabajo recibido: 7 de noviembre 2006.
}

MÉTODOS: Se analizan comparativamente dos grupos de cerdos, un grupo CONTROL ( $N=10)$ y grupo LAPAROSCÓPICO (N=10), a los que se les realiza una nefrectomía abierta o laparoscópica respectivamente. En ambos grupos se canaliza la vena yugular externa derecha y la arteria femoral y se monitoriza el GC, la PVC, la TA, las RVS (calculada mediante la fórmula RVS=lTA$P V C$ (*80/GC); estas determinaciones se realizan en los momentos: basal, 5, 30, 60 minutos y postcirugía. Mediante sonda electromagnética alrededor del vaso, se registran los FSR, FC, FP y FAH a los 30 minutos de iniciada la intervención quirúrgica.

RESULTADOS: El análisis comparativo de ambos grupos demuestra un aumento del GC en el grupo laparoscópico, cuya diferencia fue máxima a los 30 minutos $14,33+0,73$ vs $8,54+1,26 \mathrm{l} / \mathrm{min}, \mathrm{p}<0,001)$; un descenso de las RVS $11118,81+302,52$ vs 663,37 $+81,45$ dinas $\times \mathrm{s} \times \mathrm{cm}^{-5} \mathrm{p}<0,0011$ y un aumento de la TA del grupo laparoscópico $166,5+11,52$ vs $80,25+2,49 \mathrm{~mm} \mathrm{Hg}, p=0,004)$. El análisis de los flujos demostró un aumento del FAC 1 125,73 + 41,69 vs $291,70+51,52 \mathrm{ml} / \mathrm{min}, \mathrm{p}<0,0011$ y una disminución del FP $(973,67+131,70$ vs 546,83+217,53 $\mathrm{ml} / \mathrm{min}, p=0,001)$ y del FAH $(278,00+94,71$ vs $133,33+112,32 \mathrm{ml} / \mathrm{min}, p=0,03$ ) en el grupo laparoscópico. No existieron diferencias significativas en el FSR con la expansión de la volemia utilizada.

CONCLUSIONES: La nefrectomia laparoscópica condiciona un aumento del FC, posiblemente secundario al aumento del gasto cardiaco, así como un descenso de la perfusión hepática, tanto arterial como portal. Sin 
embargo, la expansión de la volemia y la reducción de la PIA a $12 \mathrm{mmHg}$ permiten mantener el FSR semejante en ambos grupos.

Palabras clave: Laparoscopia. Nefrectomía. Modelo porcino.

Summary.- OBJECTIVES: It has been demonstrated that abdominal high-pressure and the use of $\mathrm{CO} 2$ pneumoperitoneum induce changes of the cardiovascular and respiratory systems, attributable to two factors: changes of the cardiac output(CO) and hypercarbia. Other modifications derived from these facts include changes of the systemic vascular resistances (SVR), blood pressure $(B P)$, central venous pressure (CVP), vascular changes like modifications of the renal blood flow (RBF), carotid flow (CF), portal flow, and hepatic artery flow (HAF). Our objective is to analyze the hemodynamic modifications induced by pneumoperitoneum on renal blood flow, carotid flow, portal flow and hepatic artery flow in a porcine experimental model.

METHODS: We compared two groups of pigs: CONTROL group $(n=10)$ and LAPAROSCOPIC group $(n=$ 10), undergoing open or laparoscopic nephrectomy respectively. In every case, catheters were inserted into the right external jugular vein and femoral artery and cardiac output, CVP, blood pressure and systemic vascular resistances (calculated as RVS $=(\mathrm{BP} / \mathrm{CVP}) \times 80 / \mathrm{CO})$; these measurements were taken at the following times: baseline, 5, 30, $60 \mathrm{~min}$. and postoperatively. Renal blood flow, carotid flow, portal flow and hepatic artery flow were registered by means of an electromagnetic probe around the vessel 30 minutes after the start of surgery.

RESULTS: Comparative analysis shows: an increase of cardiac output in the laparoscopic group, the difference which was maximal at 30 minutes $14.33+0.73$ vs. 8 $.54+1.26 \mathrm{l} / \mathrm{min} ., p<0,0011$; a descent of the systemic vascular resistances $17118.81+302.52$ vs. $663.37+$ 81.45 dynes .s.cm5, p < 0.0011 and an increase of blood pressure $166.5+11.52$ vs. $80.25+2.49 \mathrm{~mm}$ $\mathrm{Hg}$ in the laparoscopic group. Flow analysis showed an increase of the carotid artery flow $1125.73+41.69$ vs. $291.7+51.52 \mathrm{ml} / \mathrm{min} ., p<0.0011$ and a decrease of portal flow $1973.67+131.70$ vs. 546.83+ $217.53 \mathrm{ml} / \mathrm{min} ., p=0.0011$ and hepatic artery flow $1278.00+94.71$ vs. $133.33+112.32 \mathrm{ml} / \mathrm{min} ., p=$ 0.03 in the laparoscopic group. There were no significant differences in renal blood flow with the volume expansion used.

CONCLUSIONS: Laparoscopic nephrectomy conditions an increase of carotid flow, probably secondary to the increase of cardiac output, and also a diminishment of hepatic perfusion, both arterial and portal. Nevertheless, volume expansion and the limitation of intra-abdo- minal pressure to $12 \mathrm{~mm} \mathrm{Hg}$ enable to maintain similar renal blood flow in both groups.

Keywords: Laparoscopy. Nefrectomy. Pig model.

\section{INTRODUCCIÓN}

El abordaje laparoscópico se ha erigido como una alternativa eficaz para la cirugía, ya que disminuye el trauma producido por la laparotomía, lo que redunda en una disminución del dolor postoperatorio, del tiempo de hospitalización y del tiempo para la reincorporación a la actividad normal (1).

No obstante, el neumoperitoneo necesario para la creación de un espacio que permita trabajar, implica una hiperpresión sobre la cavidad abdominal y la difusión de gas al torrente circulatorio, a través del peritoneo (2). Esto condiciona una cascada de cambios que deben ser conocidos, si se quiere realizar la técnica con seguridad.

La hiperpresión abdominal desencadena diversos efectos, independientemente del tipo de gas: un efecto simpático mediado por la vasopresina y por el sistema renina-angiotensina (3), un incremento de la presión venosa central (PVC), en tracto venoso superior, secundario a la acción compresiva sobre el territorio venoso, y una dificultad para el drenaje del tracto venoso inferior, que produce una disminución de la precarga. Esta disminución de la precarga, junto con el aumento de las resistencias vasculares, secundaria a la respuesta simpática previamente descrita, resulta en un aumento de la postcarga y una disminución del gasto cardiaco $(G C)(4,5)$.

El $\mathrm{CO}_{2}$ es absorbido a través del peritoneo debido a su alta solubilidad, generando un aumento de la presión parcial de $\mathrm{CO}_{2}$ en sangre $\left(\mathrm{PaCO}_{2}\right)$. La única forma de eliminar este $\mathrm{CO}_{2}$ excedente es a través de la ventilación pulmonar.

El aumento de la absorción de $\mathrm{CO}_{2}$ y la dificultad en su eliminación a través del pulmón, por una disminución de volúmenes pulmonares, secundaria al desplazamiento cefálico del diafragma provocado por la hiperpresión abdominal, hace que sea absolutamente necesario el ajuste continuo de la ventilación del paciente para evitar la hipercarbia. Por tanto, el factor mas importante responsable de la hipercarbia son las altas presiones intrabdominales con $\mathrm{CO}_{2}$.

La hipercarbia produce cambios hemodinámicas, no solo por afectación directa sobre el sistema cardiovascular, sino por estimulación simpática adrenal $(2,3)$. El efecto directo del $\mathrm{CO}_{2}$ sobre el miocar- 
dio provoca una disminución de la contractilidad y una sensibilización a los efectos arritmogénicos de las catecolaminas, además de vasodilatación sistémica en todo el territorio vascular.

Estos dos efectos implican a su vez numerosos cambios a nivel vascular periférico con alteraciones en la perfusión de algunos órganos, habiéndose publicado diversos trabajos que estudian los efectos del neumoperitoneo sobre la perfusión cerebral, hepática y renal $(4,6-9)$. El efecto de altas presiones intrabdominales produce cambios en los parámetros hemodinámicos y respiratorios y en la perfusión de diferentes órganos que, en pacientes sanos, son reversibles y $\sin$ aparentes secuelas.

\section{Flujo sanguíneo cerebral (FSC)}

El eco doppler transcraneal es el método no invasivo mas utilizado para medir el FSC tanto en humanos (10) como en animales (11), y el que ha demostrado reproducir con bastante fidelidad el flujo $(\mathrm{ml} / \mathrm{min}$ ) y la perfusión tisular cerebral (12), a través de la velocidad de flujo, el índice de pulsatilidad y el índice de resistencia.

EI FSC se puede afectar por cambios en la presión arterial sistémica, la glucosa, el hematocrito, la temperatura corporal, la presión parcial de oxígeno, la viscosidad sanguínea, los agentes anestésicos - muy especialmente la $\mathrm{PaCO}_{2}$, que produce relajación del músculo liso, aumentando el FSC. Se ha demostrado que por cada $\mathrm{mm}$ de $\mathrm{Hg}$ de cambio en la $\mathrm{PaCO}_{2}$ se altera el flujo cerebral en un $4 \pm 1.9 \%$ $(10,13)$. Independientemente de estos mecanismos, se ha descrito un mecanismo de redistribución del flujo sanguíneo, posiblemente mediado por catecolaminas (norepinefrina y/o vasopresina), estimulado por la distensión peritoneal, que traduce una vasoconstricción de los vasos intracraneales $(10,11,14)$.

Fujii (14) demuestra un aumento del FSC, de hasta un $30 \%$ con un neumoperitoneo de $10 \mathrm{mms}$ de $\mathrm{Hg}$, achacable a un aumento de la $\mathrm{PaCO}_{2}$ y al propio neumoperitoneo, que desaparece cuando revierte el mismo. Papadimitriou y cols. (15), insisten en la importancia de la $\mathrm{PaCO}_{2}$ sobre este fenómeno, recomendando incluso la hiperventilación profiláctica para limitar el FSC.

Se han medido niveles de malonildialdehído (MDA) en cerebro en animales sometidos a laparoscopia, no evidenciando alteraciones y demostrando que el neumoperitoneo no provoca síndrome de isquemia-reperfusión. De cualquier forma, la seguridad de la laparoscopia se debe cuestionar siempre en enfermos que no puedan tolerar mínimos cambios he- modinámicos o pequeñas alteraciones de la presión intracraneal (PIC), debido a las raras complicaciones cerebrovasculares asociadas a la laparoscopia que se han publicado $(10,16,17)$.

\section{Flujo sanguíneo hepático (FSH)}

La disminución del FSH, provocada por el neumoperitoneo sobre todo a expensas del flujo portal (FP), provocada por el neumoperitoneo ha sido constatada mediante métodos de medición directa como el doppler (18-20) o los catéteres intravasculares (21) o por métodos indirectos como la termodilución, el aclaramiento de indocianina verde $(9,22)$ o las microsferas $\gamma$ - marcadas (23). Se han realizado estudios en humanos $(19-21,24)$ y sobre modelos animales $(8,9,18,22,23,25-27)$ de los FSH, FP, así como del flujo de la arteria hepática (FAH), demostrando, en la mayoría de los casos, que con la instauración del neumoperitoneo, se produce un descenso del FSH del 35 al $84 \%$, que está en relación directa con el incremento de la PIA.

El descenso del FSH se produce, no solo por la compresión directa del $\mathrm{CO}_{2}$ sobre la pared venosa de todo el territorio venoso portal, sino por la respuesta humoral mediada por la liberación de vasopresina, estimulada por la distensión peritoneal y por la vasoconstricción producida por la hipercarbia, aunque este factor parece ser menos influyente (23), ya que el FAH prácticamente no se afecta. Richter (28) ha descrito un efecto llamado "hepatic arterial buffer response" (HABR) consistente en el aumento del FAH durante el descenso de FP generado por el neumoperitoneo, que asegura el FSH.

La importancia clínica del descenso del FSH es controvertida (23), la mayoría de los estudios no demuestran que provoque morbilidad perioperatoria, si bien la afectación de la función hepática puede reducir la síntesis proteica (fibrinógeno, albúmina...), así como el metabolismo de agentes farmacológicos, de endotoxinas, de agentes infecciosos o de células tumorales, a través de una depresión del sistema retículo endotelial de los hepatocitos, pudiendo facilitar la consecución de cuadros sépticos o a la diseminación de células tumorales.

Merece la pena destacar la incidencia de trombosis del eje espleno-portal $(29,30)$ en enfermos sometidos a esplenectomía laparoscópica por enfermedades hematológicas, entre cuyas causas destacan la trombocitosis, la sección de la vena esplénica o la disminución del flujo venoso esplácnico.

La alteración de la función hepática se ha descrito por varios autores (27) como una elevación 
transitoria de las enzimas hepáticas, sin significación clínica posterior, salvo en enfermos con función hepática preoperatoria muy deteriorada. El mecanismo de lesión hepática se produce, no solo por la disminución del flujo portal sino por una lesión de isquemia reperfusión sobre las células de Kupfer y el endotelio de los sinusoides hepáticos.

\section{Flujo sanguíneo renal (FSR)}

Se ha demostrado que la hiperpresión abdominal, producida por la laparoscopia, se asocia con una insuficiencia renal transitoria directamente relacionada con la presión intrabdominal y el tiempo de neumoperitoneo $(31,32)$. Se ha comprobado, en varios estudios, que el tipo de gas usado para el neumoperitoneo no influye en este mecanismo, recomendándose utilizar presiones bajas durante el neumoperitoneo para minimizar este fenómeno.

Existen varios mecanismos que explican este hecho:

- El descenso en el GC influye directamente sobre el FSR, ya que representa un $25 \%$ del mismo $(33,34)$, pudiendo afectar la función renal de forma secundaria. No obstante, se ha demostrado que el descenso del GC no es el único factor implicado en este hecho, puesto que aún normalizando el mismo con expansores del plasma, sigue existiendo un descenso del FSR durante el neumoperitoneo.

- El flujo sanguíneo aferente al riñón también cae hasta en un $15 \%$, por compresión del neumoperitoneo sobre la aorta y la arteria renal, cuando la PIA es superior a $10 \mathrm{~mm}$ de $\mathrm{Hg}$. Los cambios que se producen afectan por igual en el cortex y a la médula renal, retornando a valores normales cuando cesa el neumoperitoneo (35-37).

- El factor más importante implicado en este fenómeno es la compresión del parénquima renal. En este sentido Razvi y cols. (38) demuestran que, con una compresión con $15 \mathrm{~mm}$ de $\mathrm{Hg}$ sobre el parénquima renal, se objetiva una disminución de la diuresis, asociada a una disminución del filtrado glomerular $(21 \%)$ y del FSR $(26 \%)$ que no se recuperan hasta dos horas después del vaciamiento del neumoperitoneo (39). También se ha demostrado el aumento de los niveles de aldosterona, y como consecuencia de ello una disminución del sodio y un aumento del potasio en orina (40).

- Kirsch y cols. (37) describen también una compresión sobre el retorno venoso con una reducción en el flujo de la cava de hasta un $90 \%$, con presiones de neumoperitoneo relativamente usuales, demostrando que el ascenso de la PIA se asocia con disminuciones del filtrado glomerular, del FSR y de la función renal

- La obstrucción o compresión ureteral se ha sido sugerido como un mecanismo implicado en el deterioro de la función renal asociado a la laparoscopia. Sin embargo, tras los estudios de Kirsch y cols. (37), con pielogramas intravenosos y con la colocación de catéteres ureterales, se ha demostrado la ausencia de relevancia de esta situación.

Existen diversos efectos neuroendocrinos producidos por la compresión del neumoperitoneo sobre el riñón. Chiu y cols. (40) publicaron la elevación de la aldosterona durante la laparoscopia en un modelo con ratas, secundario a la activación del sistema renina-angiotensina-aldosterona desencadenado por la compresión del cortex renal. Estos cambios provocarían una disminución de la excreción fraccional de sodio y un aumento de la de potasio.

La $\beta$-D-acetilglucosaminidasa (NAG) es una proteína presente en las células tubulares renales y que se excreta en orina cuando se produce daño tubular renal. No se han evidenciado cambios en los niveles pre y postoperatorios de esta proteína tras laparoscopia (41), ni comparando el grupo de laparoscopia frente a cirugía abierta. Tampoco se objetivó relación entre los niveles de NAG y el tiempo operatorio, demostrando que no se produce lesión tubular renal significativa tras la laparoscopia, tal y como se constata en la clínica habitual, en la que tan solo en enfermos con función renal preoperatoria comprometida pueden desarrollar una insuficiencia renal que será transitoria.

La endotelina (ET) es un potente vasoconstrictor, cuyos niveles se incrementan en sangre como respuesta a la compresión de la vena renal durante el neumoperitoneo (42). Se ha demostrado que la compresión de una vena renal provoca disminución del FG y de la diuresis en ambos riñones posiblemente por la acción de esta hormona. Stowe y cols. (43) demuestran que el uso de antagonistas de los receptores de ET provoca una menor caída del FG durante la laparoscopia en ratas.

El conocimiento de todos estos cambios y su intento de corrección son necesarios para la realización de la nefrectomía laparoscópica con seguridad.

El objetivo de este trabajo es analizar las modificaciones hemodinámicas producidas por el neumoperitoneo así como sus implicaciones sobre los flujos vasculares en el sistema nervioso central, el hígado y los riñones. 


\section{MATERIAL Y MÉTODOS}

Basados en los datos obtenidos en otros estudios (44) de similares características a nuestro modelo experimental, tomando como variable indicadora el GC, y de acuerdo con los objetivos establecidos previamente, se estableció en 20 el número de animales necesarios para rechazar la hipótesis nula (ausencia de diferencias en el GC de ambos grupos a estudio), asumiendo una probabilidad del $5 \%$ de identificar una diferencia en el GC inexistente (error a) y una probabilidad del $20 \%$ de omitir (existiendo) una diferencia en el GC entre ambos grupos (error beta). El poder estadístico del experimento es, por tanto, del $80 \%$.

Se obtuvieron dos grupos de 10 cerdos, un grupo ABIERTO ( $n=10$ cerdos), utilizado como grupo control, en el que se realizó una nefrectomía por laparotomía media convencional; y un grupo LAPAROSCÓPICO: ( $n=10$ cerdos), utilizado como grupo estudio en el que la nefrectomía se realizó vía laparoscópica.

El estudio experimental se basó en el siguiente diseño:

\section{Higiene del animal}

\section{Premedicación y anestesia general del animal}

3. Laparotomía media supra e infraumbilical a 10 animales y abordaje laparoscópico transperitoneal a los 10 restantes.

4. Monitorización de variables hemodinámicas en todos los animales durante el acto quirúrgico: TAM (Tensión Arterial Media), PVC, GC y RVS (Resistencias vasculares sistémicas, calculadas mediante la fórmula $R V S=(T A M-P V C) * 80 / G C=$ dinas $x$ s $\left.\times \mathrm{cm}^{-5} 45\right)$. Los momentos de la medición, en ambos grupos, fueron: basal, a los 5, 30, 60 minutos del inicio de la cirugía $y$ tras el cierre de la piel.

5. Medidas intraoperatorias de flujo sanguíneo: FAC (Flujo arteria Carótida), FAH, FP y FSR, mediante sonda electromagnética colocada alrededor arteria carótida, arteria hepática, vena porta y en arteria renal en ambos grupos, a los 30 minutos del inicio de la cirugía.

6. Nefrectomía izquierda abierta en el grupo ABIERTO y nefrectomía izquierda laparoscópica en el grupo LAPAROSCÓPICO.

\section{Técinica anestésica:}

Tras inmovilización con lazo metálico en la corralina del animal, se administra una preparación preoperatoria con una dosis de $10 \mathrm{mg} / \mathrm{kg}$ de ketamina intramuscular, para poder canalizar una vía venosa en la oreja, por la que se mantuvo una solución de Ringer para mantener la vía. En todos los casos se administró $1 \mathrm{~g}$ de cefazolina IV en dosis única.

La inducción anestésica se realizó con 10 $\mathrm{mg} / \mathrm{kg}$ de pentotal sódico y atropina $0,01 \mathrm{mg} / \mathrm{kg}$ por vía IV. Una vez realizada la inducción, se procedió a la intubación orotraqueal, manteniendo respiración espontánea hasta su colocación en la mesa de quirófano. Posteriormente, se conectó al respirador y se mantuvo la ventilación mecánica con presión positiva intermitente, con Fracción Inspirada de Oxígeno $\left(\mathrm{FiO}_{2}\right)$ de 1, un volumen corriente minuto $(\mathrm{VM})$ inicial de $10 \mathrm{ml} / \mathrm{kg}$, ajustando este parámetro y la frecuencia respiratoria a la situación específica del momento del procedimiento quirúrgico, para intentar mantener valores de $\mathrm{CO}_{2}$ final espirado $\left(\mathrm{ETCO}_{2}\right)$ alrededor de $45 \mathrm{~mm} \mathrm{Hg}$.

El mantenimiento de la profundidad anestesica se llevó a cabo con Sevofluorano al 2-3\%, según requerimientos, junto con una perfusión continua de: $500 \mathrm{cc}$ de solución Ringer con $5 \mathrm{mg}$ de Remifentanilo, $0,15 \mathrm{mg}$ de Fentanilo y $15 \mathrm{mg}$ de Vecuronio, a una velocidad de $140 \mathrm{ml} /$ hora. Se administraron dosis adicionales de relajante muscular según necesidad.

La hidratación del animal se realizó con la perfusión de la solución de Ringer previamente mencionada (140 ml/hora), además de una infusión continua de $500 \mathrm{cc}$ de almidón (HES 130/0,4, Voluven ${ }^{T M}$ $6 \%$ ), durante la cirugía, a una velocidad de $100 \mathrm{ml} /$ $\mathrm{h}$, lo que resulta una infusión de $8 \mathrm{ml} / \mathrm{kg} / \mathrm{hora}$ de líquidos IV, ajustando, además, bolos adicionales de $50 \mathrm{cc}$, de acuerdo con los parámetros hemodinámicas registrados y las pérdidas hemáticas.

Se monitorizaron las variables hemodinámicas y respiratorias tal y como se detalla a continuación:

- Registro ECG (derivación II).

- Canalización de arteria femoral por disección para registro de TA continua.

- Canalización de vena yugular interna (Figura 1), previa disección cervical, para colocación de catéter en la arteria pulmonar, tipo OPTIQ (Abbott Lab), con el fin de obtener los valores de la PVC, el GC continuo, las presiones en arteria pulmonar y la saturación de la sangre venosa mixta (Figura 2). El valor de las RVS se calculó mediante la fórmula: $R V S=(P A M-P V C) * 80 / G C=$ dinas $x \mathrm{~s} \times \mathrm{cm}^{-5} 45$.

- Se registró la mecánica respiratoria a través de las presión de vía respiratoria, la "compliance" y la 


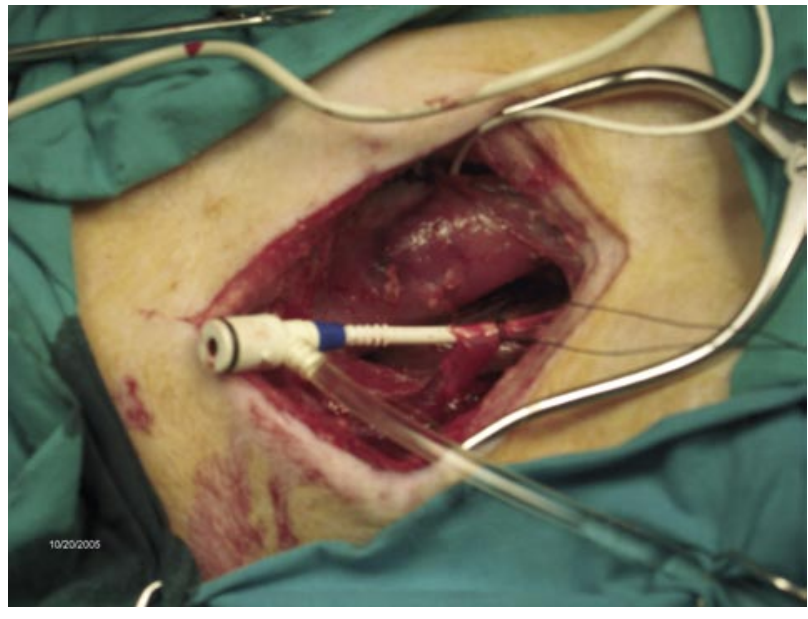

FIGURA 1. Disección y canalización de la vena yugular externa derecha.

resistencia respiratoria, así como las concentraciones de $\mathrm{O}_{2}, \mathrm{CO}_{2}$ y Sevofluorano inspirado y espirado.

- Se determinó la profundidad anestésica mediante el registro de entropía, colocando los electrodos en idéntica situación al humano.

Todos los animales se programaron para despertar después de la cirugía. Para ello se paró la perfusión de Remifentanilo, Fentanilo y Vecuronio, se mantuvo al animal sólo con Sevofluorane hasta que recuperó la respiración espontánea, con frecuencia respiratoria y volumen corriente adecuados, con control capnográfico, sin reversión del bloqueo neuromuscular. En este momento, se cortó la administración del halogenado hasta la total recuperación de reflejos del animal, seguida por la señal de Entropía.

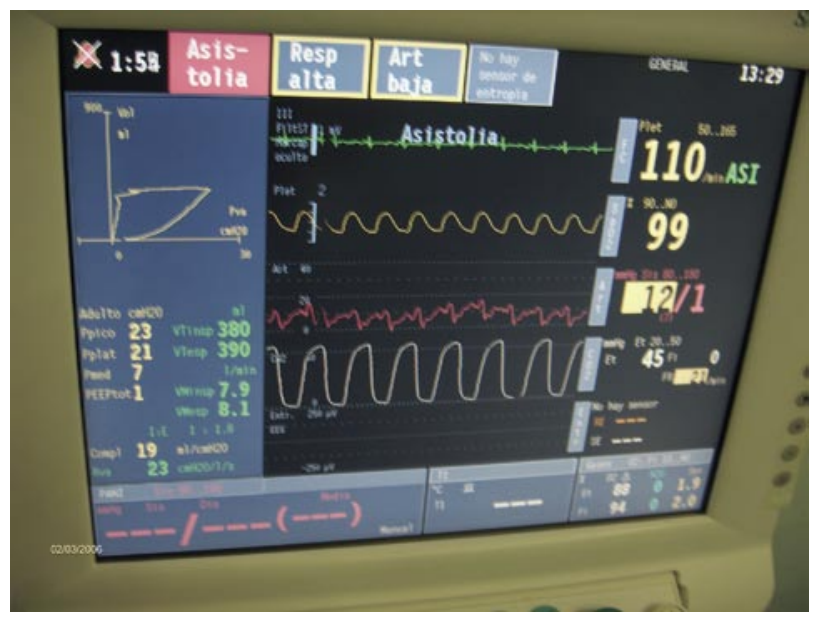

Técnica quirúrgica:

Técnica quirúrgica de nefrectomía en el grupo ABIERTO $(\mathrm{N}=10)$.

Tras la colocación y la preparación del animal expuesta anteriormente, se realizó una incisión de laparotomía media (Figura 3), abriendo toda la pared abdominal y peritoneo y accediendo al hilio hepático para disecar la vena porta y la arteria hepática para la medición del FP y FAH con sonda electromagnética.

Una vez estabilizada la medición, se procedió a la apertura del espacio laterocólico izquierdo, previa liberación de colon descendente, accediendo a retroperitoneo. Se disecó la arteria renal izquierda y se colocó el manguito de la sonda electromagnética alrededor de la misma para medición del FSR. Posteriormente se disecó de uréter para su posterior ligadura y sección. La tracción del mismo permite levantar el polo inferior renal y acceder al hilio previamente disecado, ligando y seccionando la arteria y la vena renales izquierdas, procediéndose posteriormente a la liberación y extracción del riñón. El cierre por planos de pared abdominal se llevó a cabo con polyglactil y la piel con puntos sueltos de seda.

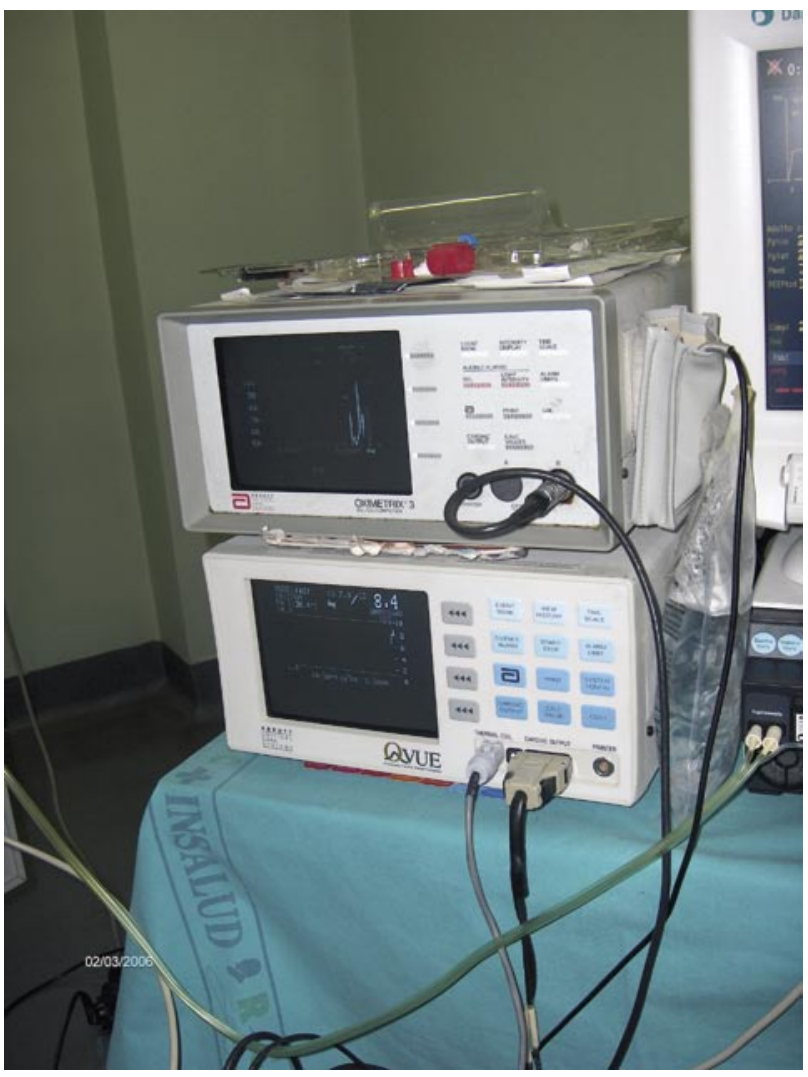

FIGURA 2. Monitorización anestésica. 


\section{Técnica quirúrgica de nefrectomía en el grupo LAPA- ROSCÓPICO ( $\mathrm{N}=10)$.}

Tras la colocación y preparación del animal en decúbito supino se realizó abordaje laparoscópico transperitoneal, previa colocación del trocar de Hasson mediante minilaparotomía de $1,5 \mathrm{cms}$ lateral a la línea mamaria, en el punto medio entre la duodécima costilla y la pala iliaca izquierdas. Se procedió a la insuflación hasta alcanzar una PIA de $12 \mathrm{~mm} \mathrm{Hg}$. El segundo trocar (10 mms) se colocó 4 $\mathrm{cm}$ lateral y caudal para manejar el instrumental con la mano derecha del cirujano y el tercer trocar de 5 $\mathrm{mm}, 4 \mathrm{~cm}$. lateral y craneal al trocar de Hasson. Se colocó otro trócar de $10 \mathrm{~mm}$ lateral a la línea mamaria derecha para el paso de la cámara durante el tiempo de aislamiento de los vasos hepáticos (Figura 4).

Tras la inspección de la cavidad peritoneal se procedió a la disección del hilio hepático, independizando la vena porta y la arteria hepática y colocando alrededor el transductor electromagnético, para medir ambos flujos sanguíneos. Posteriormente y tras la colocación del animal en decúbito lateral derecho se procedió a la apertura del espacio parietocólico izquierdo, accediendo a la celda renal y llevando a cabo la disección de la arteria renal izquierda (Figura 5), y la medición del FSR (Figura 6). A continuación, se disecó el resto del pedículo vascular renal y el uréter, procediendo a su ligadura y sección; momento en que se vació el peritoneo y se tomaron, de nuevo, todos los registros hemodinámicos y respiratorios. Tras la estabilización de los mismos, se extrajeron primero los transductores y posteriormente la pieza a través de minilaparotomía.

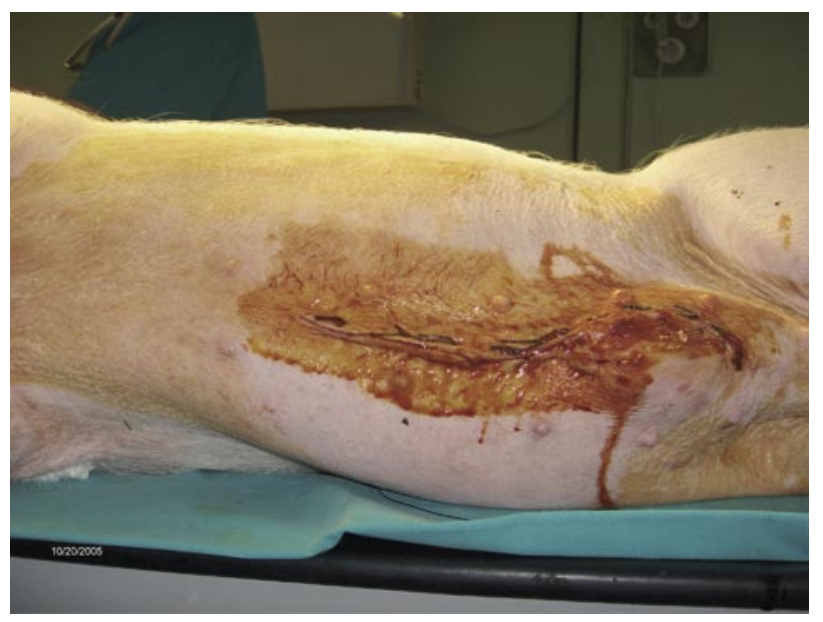

FIGURA 3. Imagen de laparotomía realizada en el grupo abierto.

\section{Método de análisis estadístico de los datos:}

Como estimador de la tendencia central de las variables se utilizó la media, y como estimador de la dispersión se empleó la desviación estándar.

Previo a la comparación de las muestras, se procedió a la exploración de los datos, mediante el análisis de los diagramas de caja de las distribuciones y a la comprobación de la normalidad de las mismas con el test de Shapiro - Wilk, que comparan la distribución observada con una esperada en caso de seguir una ley normal.

Para comparar la diferencia entre las medias observadas, se utilizó el test de la t de Student, cuando se cumplieron las condiciones de normalidad explicadas previamente (diagramas de caja sin anomalías importantes y Shapiro - Wilk no significativo) y el supuesto de homocedasticidad (test de Levene no significativo). En caso contrario, se utilizó el test no paramétrico de la U de Mann - Whitney cuando no se cumplía el supuesto de normalidad, o se obtuvieron los valores corregidos del error estándar y los grados de libertad cuando no se cumplió el supuesto de homocedasticidad.

Para el estudio de la influencia del GC y las RVS sobre los diferentes flujos, se utilizaron modelos de regresión lineal simple. En todos los casos, el GC a los 30 minutos del inicio del neumoperitoneo, actuó como variable independiente o predictora y los diferentes flujos, del mismo grupo, como variable dependiente.

Se actuó, de la misma forma, para estudiar la posible influencia de las RVS sobre los diferentes flujos.

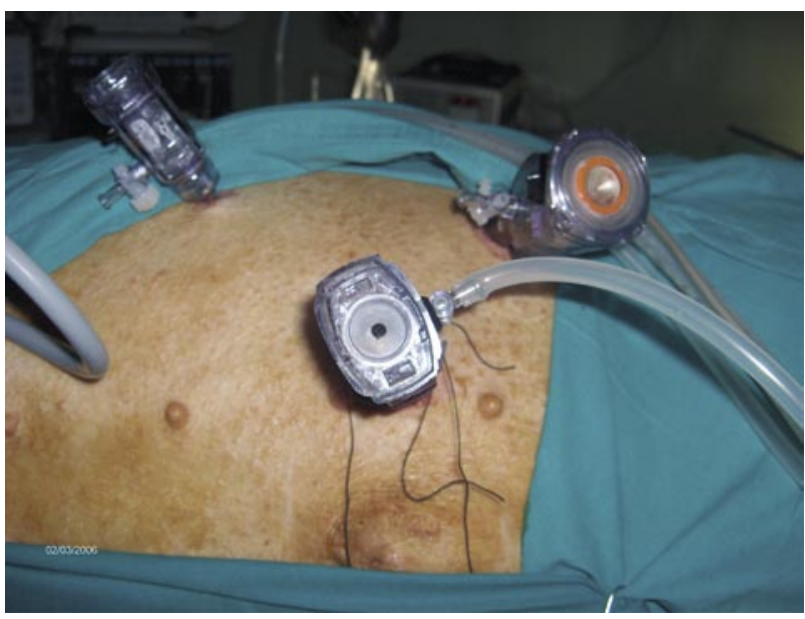

FIGURA 4. Imagen de colocación de los trócares para el acceso laparoscópico. 


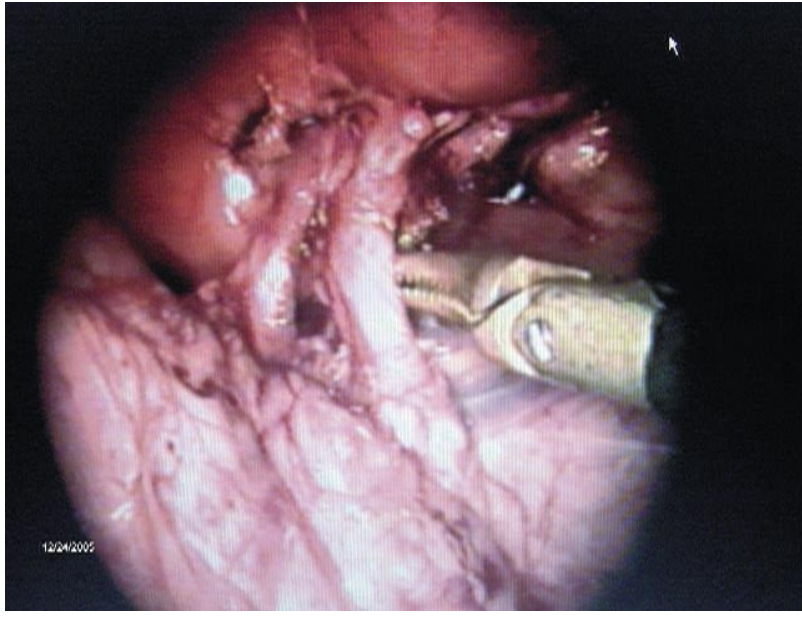

FIGURA 5. Imagen de disección de vasos renales en el grupo laparoscópico.

\section{RESULTADOS}

\section{Parámetros hemodinámicos}

El análisis comparativo del valor del GC no demostró diferencias, significativamente estadísticas, entre ambos grupos, en el momento basal, lo que demuestra la homogeneidad de los grupos. A partir de la instauración del neumoperitoneo las mediciones realizadas a los 5,30 , y 60 minutos fueron significativamente superiores en el grupo laparoscópico, haciéndose máximas a los 30 minutos $(4,33 \pm 0,73$ vs $8,54 \pm 1,26 \mathrm{l} / \mathrm{min}, \mathrm{p}<0,001)$. Tras el vaciamiento del neumoperitoneo descendieron los valores del grupo laparoscópico, acercándose de nuevo a los del grupo control, desapareciendo las diferencias previamente descritas (Figura 7).

En cuanto a la comparación del valor de la PVC, entre ambos grupos, no se demostraron diferen-

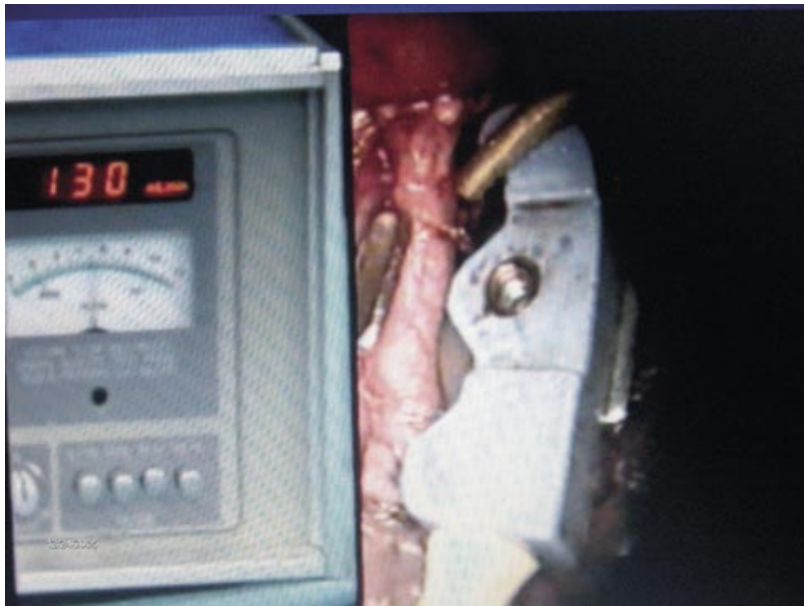

FIGURA 6. Imagen de medición de flujo de arteria renal en el grupo laparoscópico.

cias significativas ni en la determinación basal, ni en las determinaciones efectuadas durante el neumoperitoneo. Sin embargo, tras el vaciamiento, sí se demostró un aumento significativo de los valores del grupo control, frente al grupo laparoscópico $(11,44 \pm 4,30$ vs $5,33 \pm 2,64 \mathrm{~cm} \mathrm{H}_{2} \mathrm{O}, \mathrm{p}=0,008$ ) (Figura 8).

No se demostraron diferencias significativas en el análisis comparativo de los valores de TA, salvo en los valores tomados a los $30166,5 \pm 11,52$ vs $80,25 \pm 2,49 \mathrm{~mm} \mathrm{Hg}, \mathrm{p}=0,004)$ y a los 60 minutos $(67,6 \pm 10,42$ vs $78,37+3,34 \mathrm{~mm} \mathrm{Hg}, p=0,013)$, en los que se detectó un aumento significativo de la TA del grupo laparoscópico. Este aumento se normalizó inmediatamente tras la desinsuflación (Figura 9).

Los valores basales de las RVS no mostraron diferencias entre el grupo control y laparoscópico. Una vez instaurado el neumoperitoneo se evidenció
GC (L/min)

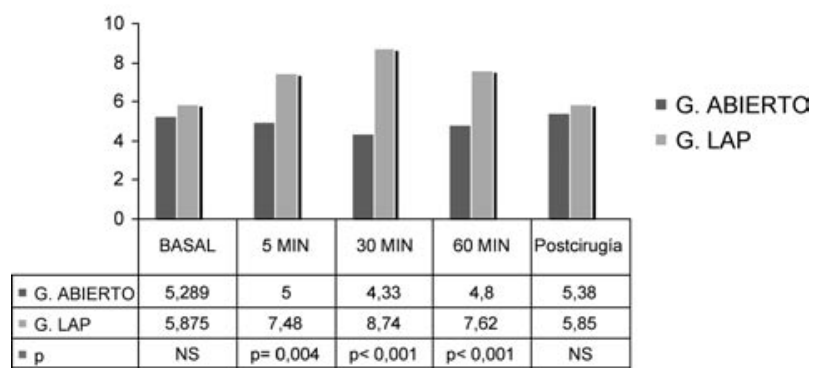

FIGURA 7. Evolución comparativa del valor GC entre los grupos abierto y laparoscópico.
PVC $\left(\mathrm{cm} \mathrm{H}_{2} \mathrm{O}\right)$

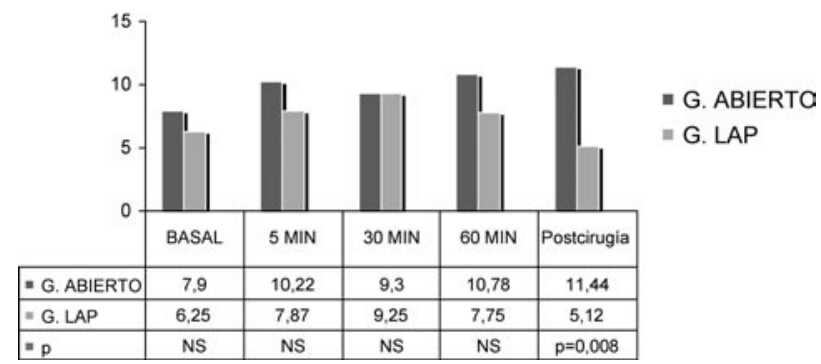

FIGURA 8. Evolución comparativa del valor PVC entre los grupos abierto y laparoscópico. 
$\mathrm{TA}(\mathrm{mm} \mathrm{Hg})$

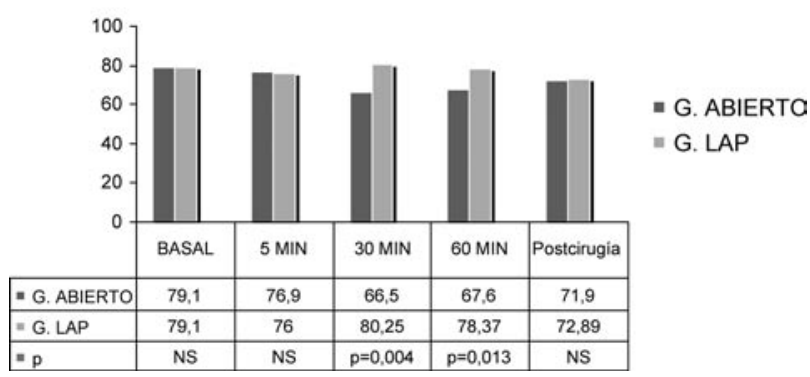

FIGURA 9. Evolución comparativa del valor TA entre los grupos abierto y laparoscópico.
RVS (dinas $x s \times \mathrm{cm}^{-5}$ )

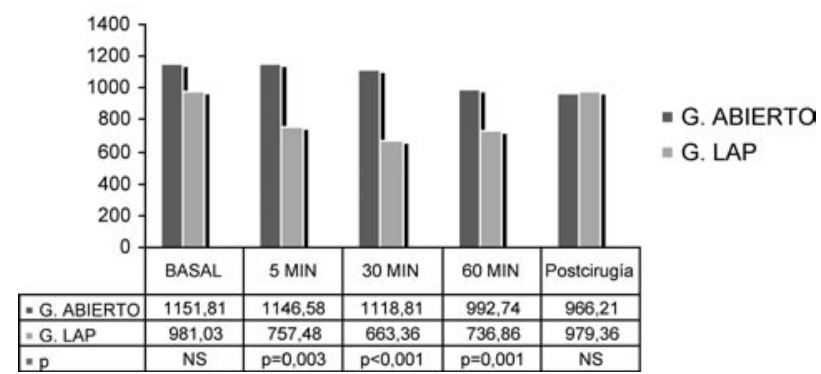

FIGURA 10. Evolución comparativa del valor RVS entre los grupos abierto y laparoscópico. un descenso significativo en la medición realizada a los 5 minutos, alcanzando su mayor diferencia a los 30 minutos $(1118,81 \pm 302,52$ vs $663,37 \pm 81,45$, dinas $\times \mathrm{s} \times \mathrm{cm}^{-5}, \mathrm{p}<0,001$ ), aunque también se demostraron diferencias significativas a los 60 minutos. No se evidenciaron diferencias tras el vaciamiento del neumoperitoneo, ya que este descenso cesó tras la desinsuflación (Figura 10).

\section{Flujos vasculares}

El análisis comparativo de los valores del flujo carotídeo, tomados a los 30 minutos del comienzo de la cirugía, entre el grupo abierto y el laparoscópico se representan en la Figura 11. Los valores

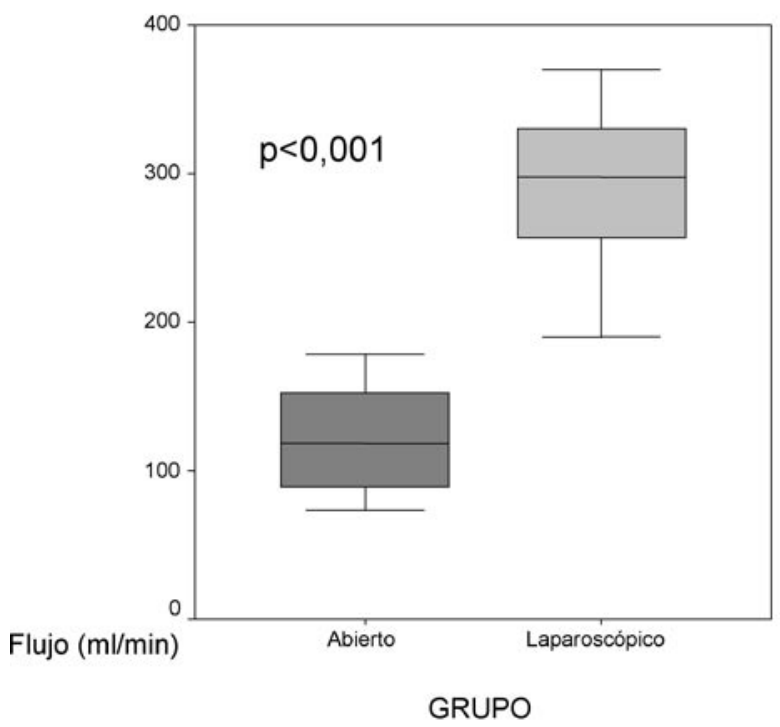

FIGURA 11. Diagrama de caja comparativo entre los valores de FAC del grupo control y laparoscópico tomados a los 30 min del comienzo de la cirugía. se tomaron, mediante sonda electromagnética, en el grupo control, al principio de la cirugía (basal). En el laparoscópico al principio de la cirugía (basal) y tras 30 minutos del inicio de la cirugía (30 minutos). Tal y como se observa en la Figura 11, se objetivó un aumento significativo del flujo carotídeo del grupo laparoscópico, respecto del grupo control, tomados a los 30 minutos del comienzo de la cirugía $(125,73$ $\pm 41,69$ vs $291,70 \pm 51,52 \mathrm{ml} / \mathrm{min}, \mathrm{p}<0,001)$. En la figura 12 , se comparan los valores de flujo carotídeo, del grupo laparoscópico, tomados en el momento basal y a los 30 minutos, siendo significativamente superiores los segundos $(209 \pm 62,8$ vs $291,70 \pm$ $51,51, p=0,009)$.

FAC grupo Laparoscópico

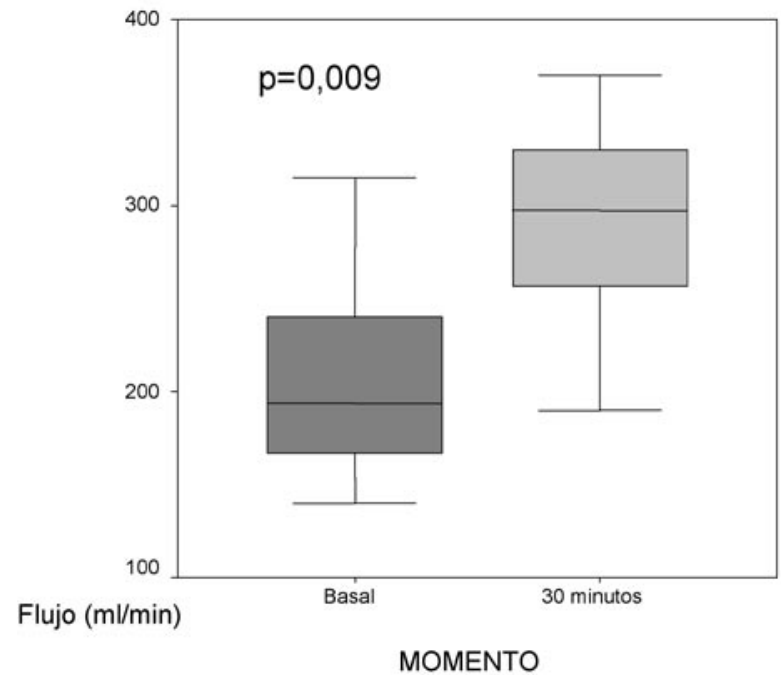

FIGURA 12. Diagrama de caja comparativo entre los valores de FAC del grupo laparoscópico tomados en el momento basal y a los 30 min del comienzo de la cirugía. 


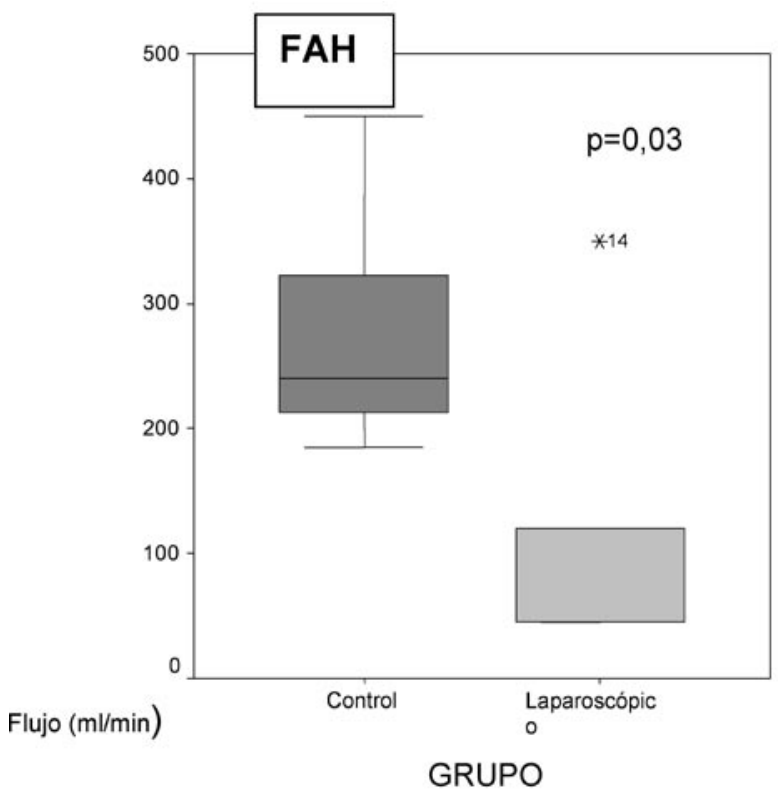

FIGURA 13. Análisis comparativo entre los valores de FAH del grupo control y laparoscópico tomados a los 30 min del comienzo de la cirugía.

El valor del flujo medido en la arteria hepática (Figura 13), a los 30 minutos del inicio de la cirugía en los dos grupos a estudio experimentó un descenso estadísticamente significativo en el grupo laparoscópico $(278,00 \pm 94,71$ vs $133,33 \pm 112,32 \mathrm{ml} / \mathrm{min}$, $p=0,03)$. En la Figura 14 observamos un descenso

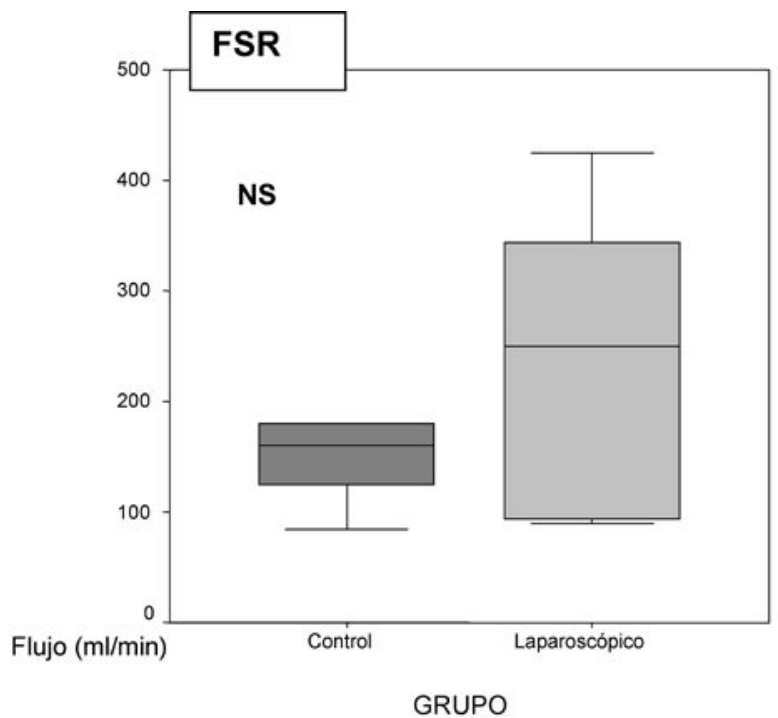

FIGURA 15. Gráfico comparativo entre los valores de FSR del grupo control y laparoscópico tomados a los 30 min del comienzo de la cirugía.

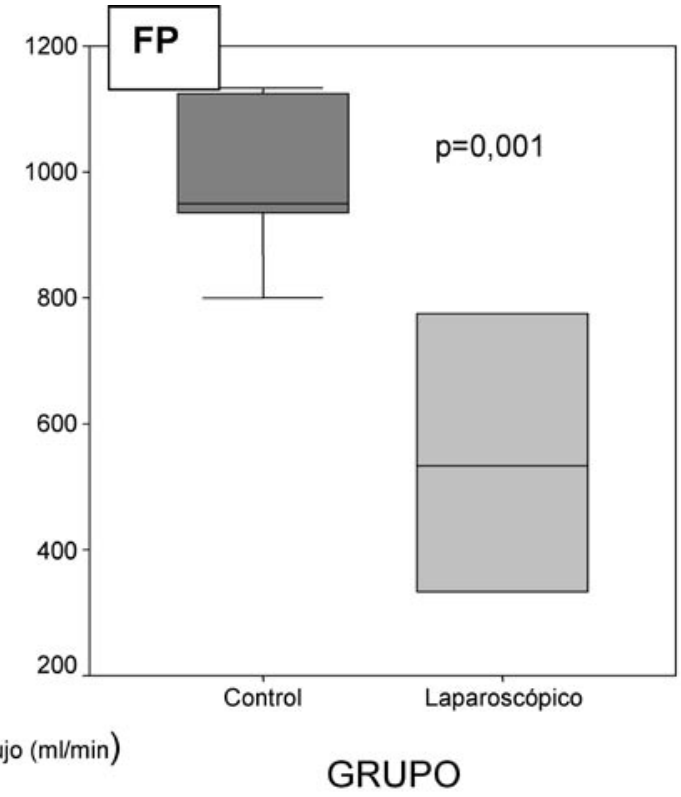

FIGURA 14. Análisis comparativo entre los valores del FP del grupo control y laparoscópico tomados a los 30 min del comienzo de la cirugía.

del FP en el grupo laparoscópico $(973,67 \pm 131,70$ vs $546,83 \pm 217,53 \mathrm{ml} / \mathrm{min}, \mathrm{p}=0,001)$, demostrándose un descenso global del flujo hepático.

El análisis estadístico comparativo de los valores de flujo sanguineo renal entre ambos grupos no

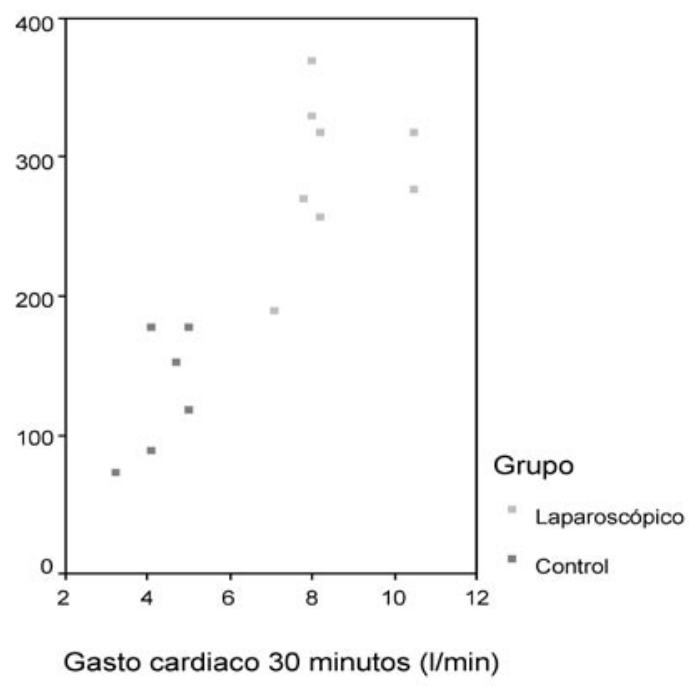

FIGURA 16. Nube de puntos que relaciona el GC, a los 30 minutos del comienzo de la cirugía, como variable independiente y el FAC, en el mismo momento, como variable dependiente. 
demostró diferencias significativas, $(234,28 \pm 141,80$ vs $183,84 \pm 95,53 \mathrm{ml} / \mathrm{min}$ ), $\mathrm{p}>0,05$ (Figura 15).

\section{Influencia del GC y las RVS sobre los flujos vascula- res.}

Los modelos de regresión lineal simple realizados para la consecución de este propósito reflejaron:

1.- Una relación estadísticamente significativa entre el valor del GC y el del FAC, a los 30 minutos de la cirugía con una $p<0,001$ y una $R 2=0,762$ (Figura 16).

2.- Una relación estadísticamente significativa inversa entre los valores de RVS y FAC, a los 30 minutos de la cirugía con una $p<0,001$ y una $R 2=0,719$ (Figura 17).

3.- Una relación inversa, estadísticamente significativa, entre el GC y el FP, a los 30 minutos de la cirugía, con una $p=0,012$ y una $R 2=0,481$ (Figura 18).

$\mathrm{Ni}$ el GC ni las RVS demostraron tener ninguna influencia (de forma independiente), sobre el FSR y el FAH. Indicando que probablemente existan otros factores más importantes implicados en estos flujos, no estudiados en este modelo.

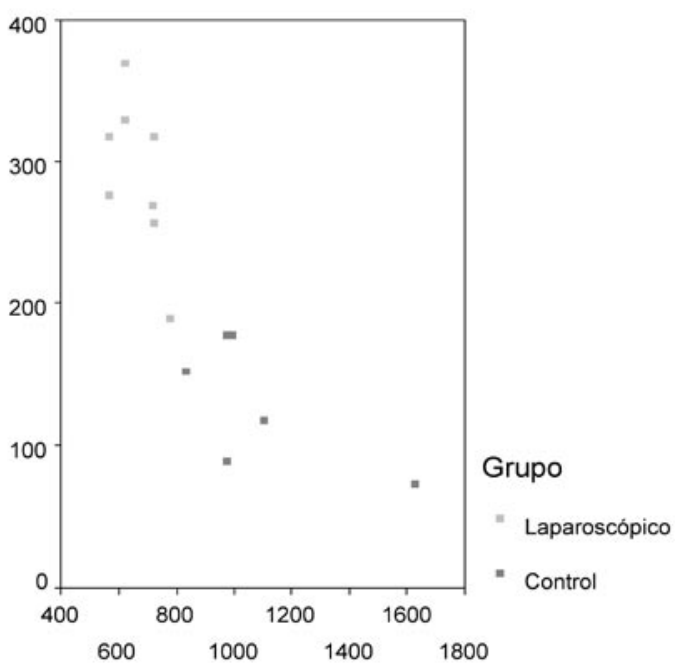

Resistencias vasculares sistémicas 30 minutos (d

FIGURA 17. Nube de puntos que relaciona el valor de las RVS, a los 30 minutos (dins $x \mathrm{~cm}^{-5}$ ) del comienzo de la cirugía, como variable independiente y el $F A C$, en el mismo momento, como variable dependiente.

\section{DISCUSIÓN}

El conocimiento de la magnitud de modificaciones hemodinámicas y su influencia sobre los diferentes flujos vasculares, en relación con la instauración del neumoperitoneo, son de vital importancia para la realización, con seguridad, de la nefrectomía laparoscópica

Para llevar a cabo nuestro estudio se eligió un modelo experimental y no clínico, por que de esta manera se tiene un control adecuado de todas las variables que intervienen en el proceso quirúrgico. Además, se escogió un animal de experimentación grande, como el cerdo, cuya anatomía cardiovascular guarda una gran similitud con el humano (44) y se realizó una nefrectomía laparoscópica, técnica urológica aceptada y realizada frecuentemente en la actualidad.

El modelo experimental propuesto semeja la realidad clínica de la nefrectomía laparoscópica, si bien algunos aspectos puede que no sean aplicables directamente en humanos. Por un lado, la presión intraabdominal de $12 \mathrm{mmHg}$ puede tener diferentes implicaciones hemodinámicas en el cerdo que en el humano. Por otro, la configuración anatómica humana, con mayor cantidad de tejido adiposo perirenal contenido en la fascia de Gerota, tiene presumible-

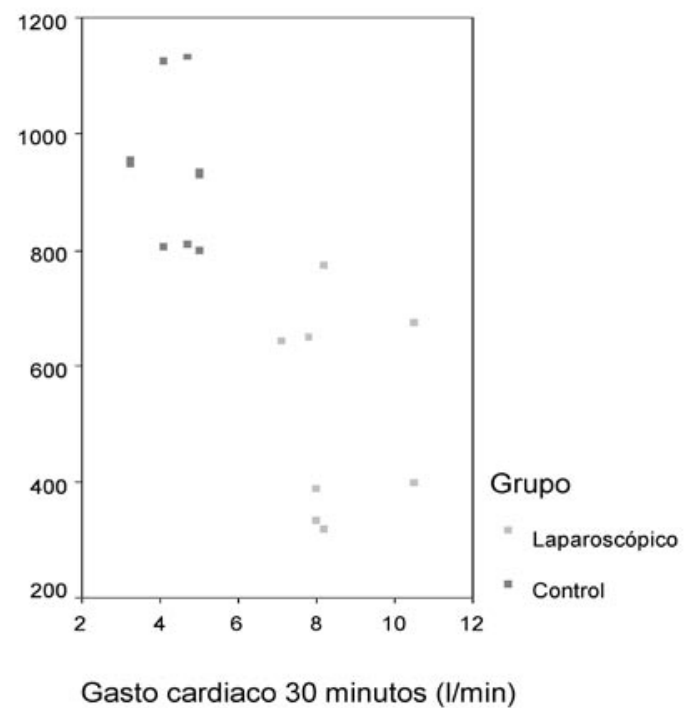

FIGURA 18. Nube de puntos que relaciona el valor del GC, a los 30 minutos del comienzo de la cirugía, como variable independiente y el FP, en el mismo momento, como variable dependiente. 
mente un efecto amortiguador de la hiperpresión abdominal sobre el parénquima renal, a diferencia del cerdo que carece de grasa perirrenal.

La exigencia de una disección y una colocación muy cuidadosa de la sonda electromagnética alrededor del vaso, así como nuestras propias dificultades técnicas en el manejo de la laparoscopia, podría haber influido mínimamente en la medición de los flujos hepáticos y renales en el grupo laparoscópico. Esta limitación la podíamos haber solventado, realizando ciclos de insuflaciones y deinsuflaciones, pero nos hubiera desviado del verdadero objetivo del trabajo, que consistía en la valoración global del abordaje laparoscópico en las condiciones más realistas posibles.

\section{Parámetros hemodinámicos}

El incremento de los valores del GC, en el grupo laparoscópico, se puede explicar, inicialmente, por una compresión de todo el territorio venoso esplácnico secundario a la hiperpresión abdominal, que posteriormente se mantuvo con valores superiores a los basales debido a un efecto simpático, producido por la hipercarbia. La reposición hidroelectrolítica intraoperatoria adecuada, que en todo momento mantuvo el volumen circulante intravascular, también pudo jugar un papel en este efecto.

Este hecho está en contraposición con los datos referidos en la literatura, en el humano, que de modo uniforme describen un descenso del GC durante el neumoperitoneo $(4,5)$. Este descenso se produce por una disminución de la precarga debido a la dificultad del retorno venoso por la compresión ejercida por el neumoperitoneo que dificulta el flujo desde los vasos venosos de capacitancia, y por un aumento brusco de las resistencias vasculares periféricas, como consecuencia de una respuesta simpática mediada por el sistema renina- angiotensina $(4,5)$.

En estudios similares en modelo porcino $(46,47)$, se observó un aumento del GC durante el neumoperitoneo semejante al descrito en nuestro estudio. En estos estudios se establece que los cambios hemodinámicas provocados por la laparoscopia son especie-dependientes y diferentes a los ocurridos en el hombre. Estas diferencias pueden estar explicadas en el cerdo por un mayor desarrollo de su tubo digestivo, lo que implica un considerable aumento de vaciamiento sanguíneo y de la precarga, si bien esto sólo explicaría el aumento inicial del GC. A partir de entonces dicho aumento, menos importante que el inicial, podría justificarse por la activación simpática inducida por el neumoperitoneo. La tendencia descendente del GC a partir de los 30 minutos apoyaría esta hipótesis.
Por tanto, parecería que la nefrectomía laparoscópica, al menos en el modelo experimental porcino condiciona un aumento significativo del GC que se hace máximo a los $30 \mathrm{~min}$ de inducido el neumoperitoneo, descendiendo posteriormente de forma lenta, pero manteniéndose todavía elevado hasta, al menos, 1 hora después, y recuperando valores basales tras la desinsuflación. La diferencia en relación al humano podría deberse a la diferencia en la anatomía digestiva, a la distinta reactividad de los vasos frente a las aminas vasoactivas liberadas durante el procedimiento y a la expansión monitorizada de la volemia.

El comportamiento de los valores de PVC del grupo laparoscópico fue parecido al del GC, presentando una tendencia ascendente hasta los 30 minutos, para descender de forma paulatina hasta el final de la cirugía. Este primer ascenso se puede explicar inicialmente por el vaciamiento esplácnico previamente mencionado. Posteriormente el descenso se explica por la compresión del neumoperitoneo sobre la vena cava inferior, y la disminución de flujo a su través hasta alcanzar los niveles basales, si bien es cierto que la pérdida sanguínea intraoperatoria podría tener alguna influencia en estas determinaciones. En el caso del grupo abierto los valores reflejados presentan una tendencia ascendente explicada por el balance hidroeléctrolítico levemente positivo diseñado para este modelo (Figura 8).

Los estudios realizados en humanos $(4,48)$ describen un aumento de la PVC secundario al vaciamiento del relleno sanguíneo de los órganos intrabdominales y al descenso del GC, provocado por el aumento de la presión intratorácica. Sin embargo, no hay que olvidar que en la clínica humana a veces los valores de PVC no reflejan de forma real ni el GC ni el volumen circulante sanguíneo efectivo.

Los modelos animales mas parecidos al modelo experimental de nuestro estudio $(46,47)$ muestran exactamente el mismo comportamiento de la PVC. Esto es, una elevación transitoria de la misma hasta la primera hora de neumoperitoneo, retornando posteriormente a los valores basales.

A diferencia del humano, en el modelo porcino, los valores de PVC parece que sí reflejan de forma real el retorno venoso. Las morfologías de las curvas del GC y de la PVC son tan parecidas que induce a pensar que el corazón porcino es capaz de asumir con relativa solvencia los volúmenes sanguíneos que le llegan bombeándolos con más facilidad que el humano. Este hecho puede estar explicado por la mayor tolerancia del abdomen a las presiones del neumoperitoneo, que conlleva una menor repercu- 
sión a nivel de presiones intratorácicas, así como las bajas presiones intrabdominales usadas en nuestro modelo (12 $\mathrm{mm} \mathrm{Hg}$ ).

La tendencia al descenso de la PVC, durante la segunda mitad de la nefrectomía laparoscópica, junto con el aumento de los valores de PVC en la nefrectomia abierta, debido a la expansión de la volemia, justificaron diferencias significativas entre los valores de la PVC de ambas técnicas tras el vaciamiento del neumoperitoneo.

Los valores de TA durante la nefrectomia abierta se mantuvieron estables con una levísima tendencia descendente no significativa. Sin embargo, los valores de TA, durante la nefrectomia laparoscópica, mostraron un leve ascenso en los primeros minutos tras el neumoperitoneo, que se mantuvo hasta la primera hora, presentando diferencias estadísticamente significativas respecto al grupo abierto a los 30 y 60 min de la insuflación. Este efecto desapareció al cesar el neumoperitoneo (Figura 9). Esta elevación transitoria de los valores de la TA, a los 30 y 60 minutos, es presumiblemente secundaria, a una respuesta adrenal simpática, aunque ésta no es demostrable en el modelo experimental que sustenta este trabajo. La valoración de niveles de actividad de renina plasmática hubiera sido una alternativa válida para poner en evidencia este aspecto, pero ésta es una circunstancia referida en la literatura tanto a nivel clínico como experimental $(4,5)$.

En una serie de 15 pacientes sometidos a colecistectomías laparoscópicas, Joris y cols. (4) refieren aumentos de la TA de hasta un 35\% durante el neumoperitoneo que estimaron secundarios a una respuesta adrenal simpática.

En los modelos experimentales de Bannenberg y Kaklamanos $(44,46,47)$ los valores de TA mostraron iguamente un incremento significativo, independientemente de los protocolos de hidratación utilizados, que en ambos casos fue Ringer lactato exclusivamente, a dosis en el caso de Bannenberg (46) de $4 \mathrm{ml} / \mathrm{Kg} / \mathrm{h}$, y en el caso de Kaklamanos (44) de $15 \mathrm{ml} / \mathrm{kg} / \mathrm{h}$. Ortega y cols. (47) no describen cambios significativos de los valores de TA, con un protocolo de hidratación a base de Ringer lactato a $1 \mathrm{cc} / \mathrm{Kg} / \mathrm{min}$.

Por tanto, parece que la nefrectomía laparoscópica en el modelo porcino condiciona un aumento de la TA presumiblemente independiente de la hidratación utilizada, y secundario a una respuesta adrenal simpática, circunstancia trasladable a la cirugía laparoscópica en humanos.
Los valores de las RVS durante la nefrectomia laparoscópica experimentaron un descenso significativo, desde unos valores basales próximos a 1000 dinas $\times \mathrm{s} \mathrm{xcm}^{-5}$ semejantes a los del grupo control, hasta alcanzar valores mínimos, próximos a 600 dinas $\times \mathrm{s} \times \mathrm{cm}^{-5}$, media hora más tarde de iniciar la insuflación. El cese del neumoperitoneo revierte este efecto de modo inmediato, volviéndose a alcanzar valores semejantes a los basales (Figura 10). Este hecho está en contraposición con los datos existentes en la literatura en el humano (4); $y$, sin embargo, en concordancia con la mayoría de estudios llevados a cabo en modelo porcino a nivel experimental $(46,47)$.

La mayoría de los trabajos realizados en humanos $(4,6)$ refieren un aumento de las RVS con el neumoperitoneo. Este aumento es probablemente secundario a la estimulación adrenérgica y justificaría, en parte, la disminución del GC descrita en la práctica clínica con las técnicas laparoscópicas en el humano. En la mayoría de los estudios realizados con cerdos $(46,47)$, se produce un descenso de las RVS, independientemente del protocolo de hidratación y de ventilación utilizada, que, tal y como se ha explicado previamente, ha sido diferente en cada caso.

En el estudio de Kaklamanos (44) se evidencia un aumento progresivo de las mismas, haciéndose máximo a los 90 minutos, con un protocolo de hidratación de $15 \mathrm{ml} / \mathrm{kg} / \mathrm{min}$ y manteniendo la normocarbia, esto es probablemente producido por la disminución del GC, evidenciada en su caso.

Probablemente, el comportamiento de las RVS, en nuestro modelo ha dependido en gran medida del valor del GC, y ha estado menos influenciado por los valores de la TA y de la PVC, ya que el comportamiento de estos dos parámetros se ha mantenido más estable y la fórmula que determina la RVS incluye estos tres parámetros.

Estos hallazgos sugerirían una menor influencia, en el modelo porcino, del efecto adrenérgico sobre las RVS, respecto del humano.

\section{Comportamiento de los flujos vasculares durante el neumoperitoneo.}

\section{Flujo de arteria carótida (FAC)}

La nefrectomía laparoscópica conlleva un mayor FAC que la nefrectomía abierta, el FAC guarda una correlación positiva con el GC y negativa con las RVS.

Winderberger y cols. (49) han estudiado el FAC en cerdos, durante el neumoperitoneo, encontrando una elevación significativa del mismo al inicio 
de la insuflación, disminuyendo posteriormente durante el transcurso del neumoperitoneo, teniendo estos cambios un comportamiento paralelo a los del GC.

Tranquart y Hänel $(12,50)$ estudiaron el FSC en animales sometidos a neumoperitoneo, sin evidenciar cambios significativos del mismo. Tampoco se han demostrado cambios de FSC durante el neumoperitoneo en humanos $(13,51)$, ya que en este caso influyen otros factores determinantes en la autorregulación del flujo cerebral como la $\mathrm{PACO}_{2} \circ$ las catecolaminas.

Debido a la autorregulación del FSC, probablemente el FAC no refleja fielmente la verdadera hemodinámica cerebral de los animales. Para evaluar de modo fiable el efecto del neumoperitoneo sobre el FSC hubieran sido necesarios otros estudios como el eco doppler transcraneal de la arteria cerebral media, que finalmente no pudimos llevar a cabo por carecer del transductor adecuado.

Es presumible que el aumento del FAC, durante el neumoperitoneo, sea secundario al incremento del GC. Este hecho está apoyado por el estudio de regresión que demuestra una relación directa del FAC con el GC e inversa con las RVS (Figuras 16 y 17 ).

La determinación del FAC tras el inicio del neumoperitoneo fue única la los $30 \mathrm{~min}$ de la insuflación), mientras que la del GC se efectuó a los 5 , 30 y $60 \mathrm{~min}$; por lo que no se pudo contar con determinaciones a los 5 y 60 min que hubiesen podido documentar de modo más fidedigno el paralelismo entre el GC y el FAC.

\section{Flujo hepático (FSH)}

La determinación de los flujos, a nivel del hígado, demostró, una menor perfusión hepática, tanto portal (FP) como arterial (FAH), en la nefrectomía laparoscópica. (Figuras 13 y 14).

Existe evidencia en humanos de que la cirugía laparoscópica conlleva un descenso del FSH, sobre todo a expensas del FP (23). La implicación clínica, de este fenómeno, no parece ser importante en enfermos sanos, si bien en ciertos casos, como los enfermos sometidos a esplenectomía laparoscópica, por enfermedad hematológica, se ha descrito un aumento en la incidencia de trombosis del eje esplenoportal $(29,30)$. Así mismo, en enfermos con función hepática comprometida, este fenómeno podría ser potencialmente trascendente.

La disminución del FSH parece dependiente, tanto en animales como en humanos, de la posición, de la hidratación y de la PIA utilizadas (25).
Igualmente, existe evidencia experimental en diferentes especies (28) que confirma este hallazgo, y en los que se constata que la reducción del flujo tiene lugar tanto a expensas del FP como del FAH. Richter y cols. (28) demostraron en ratas que durante el neumoperitoneo tenía lugar un aumento del FAH, que compensaba la reducción del FP inducida por el mismo. Este mecanismo compensatorio permite asegurar el aporte sanguíneo hepático durante la cirugía laparoscópica y se denomina "hepatic arterial buffer response".

Se debe tener en cuenta que el cerdo presenta, proporcionalmente al peso, un mayor flujo esplácnico al tener un aparato digestivo más desarrollado que el humano, esta es la razón de que en este animal se produzca una magnificación de este fenómeno, por lo que el modelo experimental elegido evalúa muy bien la influencia del neumoperitoneo en la perfusión hepática.

El fenómeno de disminución de FSH, demostrado en el presente estudio, se explica por diversos mecanismos. De un lado, el efecto compresivo del neumoperitoneo sobre la aorta y el parénquima hepático disminuiría el $\mathrm{FAH}$. De otro, el aumento de la presión intraabdominal sobre todo el territorio venoso esplácnico condiciona una disminución sobre el FP. Finalmente, la hipercapnia, producida por el neumoperitoneo, puede igualmente influir, al igual que en el resto del territorio vascular.

El aumento del GC inducido por la laparoscopia en el cerdo se ha documentado a los 30 minutos de la inducción del neumoperitoneo y asumido como secundario a las variaciones del flujo del lecho esplácnico. Sin embargo, las mediciones del FP, durante la laparoscopia parecerían no confirmar este hecho a nivel hepático. Entre las limitaciones del estudio, al principio de este apartado, se ha expuesto que, si bien cuando se realizó el grupo de nefrectomía abierta, las mediciones de FP se tomaron a los 30 minutos de la laparotomía, en el grupo laparoscópico, en cambio, la medición fue algo más tardía, dada la dificultad para disecar la porta y, por lo tanto, en medir su flujo.

Este retraso en la medición del FP en el grupo laparoscópico podría explicar la relación inversa entre el GC y el FP (Figura 18), ya que en buena lógica ambos deberían de tener una evolución paralela.

Por todo lo expuesto es presumible, que durante el neumoperitoneo, se produzca un aumento inicial de $\mathrm{FSH}$, a expensas del FP, que provoca el aumento del GC. 
Posteriormente tendría lugar un descenso del FSH, a expensas tanto del FAH como del FP no objetivándose, al menos en nuestro estudio, ningún efecto compensatorio en el aporte sanguíneo hepático.

\section{Flujo sanguíneo renal (FSR)}

La nefrectomía laparoscópica no indujo modificaciones significativas en el FSR, en comparación con la nefrectomía abierta, bajo las condiciones de mantenimiento de la volemia y de la PIA previamente mencionadas (Figura 15).

Se ha demostrado en numerosos trabajos la disminución del FSR asociada al neumoperitoneo $(35,36,40)$. Esta disminución del FSR parece provocada por varios factores como: La disminución del GC, la compresión del neumoperitoneo sobre la aorta y el pedículo vascular renal, o incluso, aunque mas dudoso, la compresión ureteral. Sin embargo, parece que el factor más importantemente implicado es la compresión sobre el parénquima renal (38).

Estudios de nuestro grupo (52), realizados en un modelo porcino de autotransplante, que comparaba la nefrectomía abierta con la laparoscópica, demostraron una disminución del FSR de hasta el 70\%, con una PIA de $15 \mathrm{~mm} \mathrm{Hg}$, y una reposición hidroelectrolítica con suero glucosalino a $10 \mathrm{ml} / \mathrm{Kg} / \mathrm{h}$.

London y cols. (53), demostraron, en un modelo experimental porcino de nefrectomía laparoscópica, la influencia de la sueroterapia en el FSR, con tres modelos diferentes de hidratación: "normovolémico" (suero salino a $3 \mathrm{ml} / \mathrm{kh} / \mathrm{h}$ ), "hipervolémico" (suero salino hipotónico a $15 \mathrm{ml} / \mathrm{kg} / \mathrm{h}$ ), e "hipertónico" (misma hidratación que grupo normovolémico añadiendo suero salino al 7,5\% a $1,2 \mathrm{ml} / \mathrm{kg} / \mathrm{h}$ ). Mientras que en el grupo "normovolémico" se objetivaba un descenso del FSR hasta del $70 \%$, en los otros dos grupos, parecía minimizarse este efecto, consiguiendo incluso en el grupo "hipertónico" ascensos moderados del FSR.

Por otro lado, Junghans y cols. (25), en un modelo porcino, en el que estudiaron la influencia de diferentes gases, diferentes posiciones y diferentes PIAS, en flujos sanguíneos hepático y renal, demostraron la importancia de la PIA sobre el FSR, constatando que PIAS mayores de $12 \mathrm{~mm}$ de hg provocaban afectaciones en los FSR de hasta el $25 \%$.

Los poderes de expansión de la volemia de las diferentes soluciones de infusión intravenosas juegan un papel importante en el mantenimiento del volumen circulante efectivo, que a su vez asegura la estabilidad hemodinámica necesaria para mantener unos flujos viscerales adecuados. En este sentido, es destacable que el poder de expansión de volumen de la solución de Ringer lactato o del suero salino isotónico, que es de 0,20 , es decir que a los, aproximadamente 20 minutos de la infusión de los mismos, tan sólo quedará el $25 \%$ de la solución infundida en el espacio intravascular. El hidroxietilalmidón (HES 130/0,4 al 6\%) es una solución coloide sintética con una capacidad de unión al agua próxima a los $20-30 \mathrm{ml} / \mathrm{g}$. Esta propiedad le confiere un poder de expansión de volumen, en torno a 0,8 , circunstancia que permite mantener un mayor volumen circulante efectivo, durante mas tiempo (54).

Por todo lo expuesto anteriormente, se decidió realizar la reposición hidroelectrolítica con una perfusión de la solución de Ringer a $140 \mathrm{ml} / \mathrm{h}$, además de una infusión continua de almidón, durante la cirugía, a una velocidad de $100 \mathrm{ml} / \mathrm{h}$, lo que equivale a una infusión total de $8 \mathrm{ml} / \mathrm{kg} /$ hora de líquidos IV, ajustando, además, bolos adicionales de $50 \mathrm{ml}$, de acuerdo con los parámetros hemodinámicos registrados y las pérdidas hemáticas.

El presente trabajo experimental no objetivó diferencias, entre los FSR, durante los procedimientos de las nefrectomías abierta o laparoscópica. Este hecho podría estar condicionado por dos factores. El primero, por el mantenimiento del valor de la PIA de $12 \mathrm{~mm} \mathrm{Hg}$, que minimiza los efectos adversos del neumoperitoneo, permitiendo trabajar con cierta comodidad. El segundo factor es el uso de un $40 \%$ de macromoléculas de almidón en el volumen infundido, que permite alcanzar un volumen circulante efectivo suficiente para el mantenimiento de un FSR aceptable, sin favorecer el paso de excesivos líquidos al espacio intersticial. La monitorización y vigilancia exhaustiva de los parámetros hemodinámicas es, sin duda, otro factor a tener en cuenta.

La ausencia de asociación significativa entre el GC y el FSR, sugiere la presencia de otros factores implicados en la hemodinámica renal. Chiv y cols. (36), en un modelo porcino, en el que estudia el efecto de la PIA sobre la circulación renal describe un efecto shunt del flujo sanguíneo del cortex a la médula renal, probablemente producido por factores neurohumorales.

Por tanto, la nefrectomía laparoscópica condicionaría una disminución del FSR que podría ser atenuada aumentando o estabilizando el volumen circulante efectivo con expansores del plasma como el hidroxietilalmidón. A pesar de mejorar el FSR, podrían existir otros cambios hemodinámicos intrarrenales, presumiblemente secundarios a factores neurohumorales sobre los cuales es difícil de establecer el efecto que tendría la expansión de la volemia. 


\section{CONCLUSIONES}

El comportamiento de los diferentes flujos durante el neumoperitoneo y en las condiciones previamente descritas, es dispar. Tanto el FAC como el FSR parecen haber sido modificados por el ascenso del GC y el descenso de las RVS, consecuencia del aumento del volumen circulante efectivo. Sin embargo, la reducción de los FAH y FP, inducidas por el neumoperitoneo estarían menos influenciadas por la reposición hidroelectrolítica. Las causas de este comportamiento dispar de los distintos lechos vasculares, así como del incremento de la TA, podrían estar en relación con mecanismos de autorregulación mediados por factores neurohumorales a nivel periférico.

\section{BIBLIOGRAFIA y LECTURAS RECOMENDADAS ( ${ }^{*}$ lectura de interés $y^{* *}$ lectura fundamental)}

1. TAMALES, S.: "Avances en cirugía laparoscópica”. An. R. Acad. Nac. Med., 114: 383, 1997.

2. HSIEH, L.H.: "Laparoscopic cholecystectomy for patients with chronic obstructive pulmonary disease”. Laparoendosc. Adv. Surg. Tech., 13: 5, 2003.

3. NEUDEEKER, J.; SAUERLAND, S.; NEUGEBAUER, M. y cols.: "The EAES clinical practice guidelines of pneumoperitoneum for laparoscopic surgery”. Surg. Endosc., 16: 1121, 2002.

*4. JORIS, J.L.; NOIROT, D.P.; LEGRAND, M.J. y cols.: "Hemodynamyc changes during laparoscopic cholecystectomy". Anest. Analg., 75: 1067, 1993.

5. O' LEARY; HUBBARD, K.; TORMEY, W. y cols.: "Laparoscopic cholecystectomy: Haemodynamic and neuroendocrine responses after pneumoperitoneum and changes in position". Br. J. Anaesth., 76: 640, 1996.

*6. GUTT, C.N.; ONIU, T.; MEHRABI, A. y cols.: "Circulatory and respiratory complications of carbon dioxide insufflation”. Dig. Surg., 21: 95, 2004.

*7. FUJII, Y.; TANAKA, H.; TSURUOKA, S. y cols.: "Middlecerebral arterial blood flow velocity increases during laparoscopic cholecystectomy". Anesth. Analg., 78: 80, 1994.

8. KLOPFENSTEIN, C.E.; MOREL, D.R.; CLERGUE, F. y cols.: "Effects of abdominal CO2 insufflation and changes of position on hepatic blood flow in anesthetized pigs". Am. J. Physiol., 275: 900, 1998.

9. SALA-BLANCH, X.; FONTANALS, J.; MARTINEZ-PALLI, G. y cols.: "Effects of carbon dioxi- de vs helium pneumoperitoneum on hepatic blood flow". Surg. Endosc., 12: 1121, 1998.

10. HUETTEMANN, E.; TERBORG, C.; SAMIR, G. y cols.: "Preserved $\mathrm{CO} 2$ reactivity and increase in middle cerebral arterial blood flow velocity during laparoscopic surgery in children". Anesth. Anal., 94: 255, 2002.

11. ERKAN, N.; GOKMEN, A.Y.; GOKTAY, G. y cols.: "Effects of $\mathrm{CO}_{2}$ pneumoperitoneum on the basilar artery. An experimental study in rabbits". Surg. Endosc., 15: 806, 2001.

12. TRANQUART, F.; BERSON, M.; BODARD, S. y cols.: "Evaluation of cerebral blood flow in rabbits with transcranial Doppler sonography: first results". Ultrasound Med. Biol., 17: 815, 1991.

13. KETY, S.S.; SCHMIDT, C.F.: "The effects of altered arterial tensions of carbon dioxide and oxygen on cerebral blood flow and cerebral oxygen consumption of normal young men". J. Clin. Invest., 27: 484, 2001.

14. FUJII, Y.; TANAKA, H.; TSURUOKA, S. y cols.: "Middle cerebral arterial blood flow velocity increases during laparoscopic cholecystectomy". Anesth. Analg., 78: 80, 1994.

15. PAPADIMITROU, L.S.; LIVANIUS, S.H.; MOKA, E.G. y cols.: "Cerebral blood flor velocity alterations Ander two different carbon dioxide management strategies, during sevoflurane anesthesia in gynaecological laparoscopic surgery". Neurol. Res., 25: 361, 2003.

16. BONATSOS, G.; LEANDROS, E.; DOURAKIS, N. y cols.: "Laparoscopic cholecystectomy: intraoperative findings and postoperative complications". Surg. Endosc., 9: 889, 1995.

17. MULLER, K.; MEIER-PETER, A.; PISCOL, C. y cols.: "Localized cerebral circulatory disorder as an unusual complication following laparoscopy". Med. Klin., 62: 1790, 1967.

*18. GUTT, C.N.; SCHMANDRA. T.C.: "Portal venous flow during $\mathrm{CO} 2$ pneumoperitoneum in the rat". Surg. Endosc., 13: 902, 1999.

19. TAKAGI, S.: "Hepatic and portal vein blood flow during carbon dioxide pneumoperitoneum for laparoscopic hepatectomy”. Surg. Endosc., 12: 431, 1998.

20. SCHILLING, M.K.; KRÄHENBÜHL. L. y cols.: "Splanchnic microcirculatory changes during $\mathrm{CO}_{2}$ laparoscopy". J. Am. Coll. Surg., 184: 378, 1997.

21. ODEBERG, S.; LJUNGQVIST, O.; SOLLEVI, A. y cols.: "Pneumoperitoneum for laparoscopic cholecystectomy is not associated with compromise of splanchnic circulation". Eur. J. Surg., 164: 843, 1998.

22. TUNON, M.J.; GONZALEZ, P.; JORQUERA, F. y cols.: "Liver blood flow changes during laparoscopic surgery in pigs. A study of hepatic indocyanine green removal". Surg. Endosc., 13: 668, 
1999.

23. SCHÄFER,M.;SÄGESSER,H.;KRÄHENBÜHL, L. y cols.: "Liver and splanchnic hemodynamic changes in rats during laparoscopy". Surg. Endosc., 14: S216, 2000.

*24. JAKIMOWICZ, J.; STULTIENS, G.; SMULDERS, F. y cols.: "Laparoscopic insufflation of the abdomen reduces portal venous flow". Surg. Endosc., 12: 129, 1999.

**25. JUNGHANS, T.; BOHM, B.; GRUNDEL, K. y cols.: "Does pneumoperitoneum with different gases, body positions and intraperitoneal pressures influence renal and hepatic blood flow?'. Surgery, 121: 206, 1997.

26. RASMUSSEN, I.B.; BERGGREN, U.; ARVIDSSON,D. y cols.: "Effects of pneumoperitoneum on splanchnic hemodynamics: an experimental study in pigs". Eur. J. Surg., 161: 819, 1995.

27. WINDBERGER, U.B.; AUER, R.; KEPLINGER, F. y cols.: "The role of intra-abdominal pressure on splanchnic and pulmonary hemodynamic and metabolic changes during carbon dioxide pneumoperitoneum". Gastrointest. Endosc., 49: 84, 1999.

28. RICHTER, S.; VOLLMAR, B.; MUCKE, I. y cols.: "Hepatic arterio-portal venous shunting guarantees maintenance of nutritionalmicrovascular blood supply in hepatic arterial buffer responseof rat livers". J. Physiol., 531: 192, 2001.

29. PIETRABISSA, A.; MORETTO, C.; ANTONELLI, G. y cols.: "Thrombosis in the portal venous system after elective laparoscopic splenectomy". Surg. Endosc., 18: 1140, 2004.

30. HARRIS, W.; MARCACCIO, M. y cols.: "Incidence of portal vein thrombosis after laparoscopic splenectomy". J. Can. Chir., 48, 2005.

31. GULER, C.; SADE, M.; KIRKALI, Z. y cols.: "Renal effect of carbon dioxide insufflation in rabbit pneumoperitoneum model". J. Endourol., 12: 367, 1998.

32. KHEIRABADI, B.; TUTHILL, D.; PEARSON, R. y cols.: "Metabolic and hemodynamic effects of $\mathrm{CO} 2$ pneumoperitoneum in a controlled hemorrhage model". J. Trauma., 50: 1031, 2001.

33. SAFRAN, D.B.; ORLANDO, R. y cols.: "Physiologic effects of pneumoperitoneum". Am. J. Surg., 167: 281, 1994.

34. KASHTAN, J.; GREEN, J.F.; PARSONS, E.Q. y cols.: "Hemodynamic effects of increased abdominal pressure". J. Surg. Res., 30: 249, 1981.

35. CHIU, A.W.; CHANG, L.S.; BIRKETT, D.H. y cols.: "The impact of pneumoperitoneum, pneumoretroperitoneum, and gasless laparoscopy on the systemic and renal hemodynamics". J. Am. Coll. Surg., 181: 397, 1995.

*36. CHIU, A.W.; AZADZOI, K.M.; HATZICHRIS-
TOU, D.G. y cols.: "Effects of intrabdominal pressure on renal tissue perfusion during laparoscopy". J. Endourol., 8: 99, 1994.

37. KIRSCH, A.J.; HENSLE, T.W.; CHANG, D.T. y cols.: "Renal effects of $\mathrm{CO} 2$ insufflation: Oliguria and acute renal dysfunction in a rat pneumoperitoneum". Urology, 43: 453, 1994.

*38. RAZVI, H.A.; FIELDS, D.; VARGAS, J.C. y cols.: "Oliguria during laparoscopic surgery: Evidence for direct renal parenchymal compression as an etiologic factor". J. Endourol., 10: 1, 1996.

39. HERMAN, F.G.; WINTON, R.F.: "The interaction of intrarenal and extrarenal pressures". J. Physiol., 87: 77, 1936.

40. CHIU, A.W.; CHANG, L.S.; BIRKETT, D.H. y cols.: "Changes in urinary output and electrolytes during gaseous and gasless laparoscopy". Urol. Res., 24: 361, 1996.

41. MICALI, S.; SILVER, R.I.; KAUFMAN, H.S. y cols.: "Measurement of urinary $\mathrm{N}$-acetyl-beta-Dglucosaminidase to assess renal ischemia during laparoscopic operations”. Surg. Endosc., 13: 503, 1999.

42. HAMILTON, B.D.; CHOW, G.K.; STOWE, N.T. y cols.: "The effect of renal vein compression on renal function: A canine model for laparoscopic surgery”. J. Endourol., 11: S53, 1997.

43. STOWE, N.T.; SUNG, G.T.; SOBEL, J.J. y cols.: "Endothelin antagonist attenuation pneumoperitoneum-induced fall in GFR in a rat model". J. Endourol., 12: 97, 1998.

*44. KAKLAMANOS, I.G.; CONDOS, S.; MERRELL, R.C. y cols.: "Time-related changes in hemodynamic parameters and pressure-derived indices of left ventricular function in a porcine model of prolonged pneumoperitoneum". Surg. Endosc., 14: 834, 2000.

45. CLARK, E.B.; VAN MIEROP, L.H.S.: "Development of the cardiovascular system". Heart disease in infants, children and adolescents , 4 ed. Edited by Adams FH, Emmanouilides GC, and Eimenschneider TA, Baltimore, 2, 1989.

**46. BANNENBERG, J.; RADEMAKER, B.; FROELING, F. y cols.: "Hemodynamics during laparoscopic extra-and intraperitoneal insufflation. An experimental study”. Surg. Endosc., 11: 911, 1997.

**47. ORTEGA, A.; RICHMAN, M.; HERNANDEZ, M. y cols.: "Inferior vena caval blood flow and cardiac hemodynamics during carbon dioxide pneumoperitoneum”. Surg. Endosc., 10: 920, 1996.

48. KOIVUSALO, A.M.; LINGREN, L. y cols.: "Effects of carbon dioxide pneumoperitoneum for laparocopic cholecystectomy". Act. Anaesthesiol. Scand., 44: 834, 2000.

49. WINDERBERGER, U.; SIEGL, H.; FERGUS- 
SON, J.G. y cols.: "Hemodynamic effects of prolonged abdominal insufflation for laparoscopic procedures". Gastrointest. Endosc., 41: 121, 1995.

50. HÄNEL, F.; BLOBNER, M.; BOGDANSKI, R. y cols.: "Effects of Carbon Dioxide Pneumoperitoneum on cerebral Hemodynamics in Pigs". J. Neurosurg. Anesth., 13: 222, 2006.

51. HUETTEMANN, E.; TERBORG, C.; SAMIR, G. y cols.: "Preserved $\mathrm{CO} 2$ Reactivity and Increase in Middle Cerebral Arterial Blood Flow Velocity During Laparoscopic Surgery in Children". Anesth. Anal., 94: 255, 2002.

**52. LINARES QUEVEDO, A.I.: "Estudio comparativo de la función del transplante renal con donante vivo mediante nefrectomía convencional y laparoscópica. Influencia del síndrome de isquemia repercusión. Modelo experimental". Madrid, 2005.

**53. LONDON, E.; HUNG, S.; NEUHAUS, A. y cols.: "Effect of Intravascular Volume Expansion on Renal Function During Prolonged CO2 Pneumoperitoneum”. Ann. Surg., 231: 195, 2000.

*54. BOLDT, J.; SUTTNER, S: "Plasma substitutes". Minerva Anestesiol., 71: 741, 2005. 